\title{
INFLUENCE OF RETRIEVED HIP-AND KNEE-PROSTHESIS BIOMATERIALS ON MICROBIAL DETECTION BY SONICATION
}

\author{
S. Karbysheva ${ }^{1,2,3}$, L. Grigoricheva ${ }^{2}$, V. Golnik ${ }^{2}$, S. Popov ${ }^{2}$, N. Renz ${ }^{1}$ and A. Trampuz ${ }^{1,3, *}$ \\ ${ }^{1}$ Centre for Musculoskeletal Surgery, Charité - Universitätsmedizin Berlin, corporate member of \\ Freie Universität Berlin, Humboldt-Universität zu Berlin and Berlin Institute of Health, Berlin, Germany \\ ${ }^{2}$ Federal Centre of Traumatology, Orthopaedics and Arthroplasty, Barnaul, Russia \\ ${ }^{3}$ Berlin-Brandenburg Centre for Regenerative Therapies (BCRT), Berlin, Germany
}

\begin{abstract}
Microorganisms' ability to adhere and form a biofilm differs among biomaterials; however, clinical data are conflicting. Microbial adherence and biofilm formation on different biomaterials of explanted joint prosthesis components were investigated. Consecutive patients with explanted joint prosthesis were prospectively included. The bacterial load dislodged from retrieved prosthetic components was evaluated qualitatively and quantitatively in sonication-fluid cultures. For comparison between groups, one-way ANOVA and Wilcoxon signed-rank test were used. A total of 112 components originating from 58 knee and 54 hip prostheses were retrieved from 40 patients. Components were made of titanium alloy in 42 cases, cobalt-chromium alloy in 38 and polyethylene in 32 . Bacteria in sonication-fluid cultures grew in all polyethylene components (100\%). Larger bacterial counts were found on polyethylene than on titanium $(p<0.013)$ or cobalt-chromium alloy ( $p=0.028$ ). Coagulase-negative Staphylococci, Staphylococcus aureus and Streptococcus species were most commonly isolated. In conclusion, polyethylene showed larger biofilm burden than metal alloys, indicating their higher microbial adhesion affinity in vivo.

Moreover, bacterial counts were larger after sonication of polyethylene liners than of metal alloys, suggesting intrinsic differences in the ability of microorganisms to form biofilms on various biomaterials. Polyethylene liners allowed the diagnosis of prosthetic joint infections (PJIs) in all investigated cases, suggesting that sonication of polyethylene liners rather than of the complete prosthesis was sufficient for pathogen detection in PJIs.
\end{abstract}

Keywords: Biofilm, arthroplasty, polyethylene, metal, sonication, biomaterials.

*Address for correspondence: Andrej Trampuz, MD, Charité - Universitätsmedizin Berlin, Centre for Musculoskeletal Surgery, Mittelallee 4, D-13353 Berlin, Germany.

Telephone number: +49 30450615073 Fax number: +49 30450552987 Email: andrej.trampuz@charite.de

Copyright policy: This article is distributed in accordance with Creative Commons Attribution Licence (http://creativecommons.org/licenses/by-sa/4.0/).

\section{Introduction}

The key phenomenon in the pathogenesis of a prosthetic joint infection (PJI) is the attachment of microorganisms to the implant surface and the subsequent biofilm formation. In this situation, conventional diagnostic methods (such as synovial fluid and periprosthetic tissue culture) have a limited sensitivity, reported at 60-70\% (Evangelopoulos et al., 2013; Trampuz et al., 2007). The culture sensitivity is especially limited in chronic infections, typically associated with low microbial burden, and in patients previously receiving antibiotics (Puig-Verdie et al., 2013).

Modern diagnostic technologies increase the sensitivity of peri-implant tissue and synovial fluid cultures. For example, culturing specimens in blood culture bottles demonstrates superior diagnostic performance as compared to conventional agar plate cultures (Font-Vizcarra et al., 2012; Peel et al., 2016). However, most microbiology laboratories use the agar plate method, with limited sensitivity ranging around 60-82 \% (Evangelopoulos et al., 2013; Larsen et al., 2012; Trampuz et al., 2007). As the 
key phenomenon in the pathogenesis of implantassociated infection is the biofilm formation on the implant surface, sonication of explanted prostheses is superior when compared to periprosthetic tissue culture (Larsen et al., 2012; Trampuz et al., 2007).

The influence of the biomaterial type on the biofilm formation is controversial. Experimental animal studies suggest that microbial adherence and biofilm formation depend on the type of material (Cordero et al., 1994; Cordero et al., 1996; Petty et al., 1985). However, clinical studies show conflicting data regarding microbial adherence to materials. Lass et al. (2014), in a study including hip prosthesis components, show that polyethylene liners and prosthesis heads have larger bacterial loads than other components. Gomez-Barrena et al. (2012), in a study investigating retrieved hip and knee components, observe no differences in bacterial adherence to individual biomaterials. Bacterial adherence depends on the type of microorganism rather than the type of biomaterial.

To provide insights into the role of biomaterials, such as polyethylene, titanium and cobalt-chromium alloy, the influence of the material type on the biofilm formation was investigated. For this purpose, the biofilm was investigated by sonication-fluid culture of retrieved hip and knee prostheses. Such findings might help further optimising microbiological diagnosis from retrieved implants using the sonication procedure.

\section{Materials and Methods}

\section{Study design}

The study was conducted at the Federal Centre of Traumatology, Orthopaedics and Arthroplasty in Barnaul (Russia), providing advanced specialty care to a population of 7.3 million inhabitants. Data were extracted from electronic medical charts. The study protocol was submitted to the local Ethics Committee, which considered the microbial investigation of retrieved prosthetic components as a part of a routine diagnostic procedure and waived the need for informed consent.

\section{Study population}

Consecutive patients with complete or partial prosthesis explantation due to chronic PJI (see definition below), in which at least one component of the prosthesis showed bacterial growth, were included in the present prospective cohort study, performed from January 2015 until December 2016. Patients with acute PJI - i.e. symptom duration less than 4 weeks or infection manifesting within 1 month after implantation, receiving antibiotics prior to surgery, in whom sonication of the retrieved implant was not performed or obvious contamination of the explanted material occurred during surgery, transport to or processing in the microbiology laboratory - were excluded.

\section{Definitions}

PJI was diagnosed according to the working criteria of the European Bone and Joint Infection Society (EBJIS), as done in several studies (Akgun et al., 2018; Akgun et al., 2017; Morgenstern et al., 2018; Renz et al., 2017; Renz et al., 2018a; Renz et al., 2018b; Sigmund et al., 2018). Accordingly, PJI is diagnosed when one or more of the following criteria are met: (i) presence of sinus tract or macroscopic purulence; (ii) positiveinflammation histopathology of periprosthetic tissue, defined as $\geq 23$ granulocytes per 10 high-power fields (i.e. type II or III according to Krenn et al., 2014); (iii) increased synovial fluid leukocyte count, defined as $>2000$ leukocytes/ $\mu \mathrm{L}$ or $>70 \%$ granulocytes; (iv) positive synovial fluid, periprosthetic tissue or sonication-fluid culture. Sonication culture was considered positive if $\geq 50$ colony-forming units (CFU)/mL were detected, except for Staphylococcus aureus ( $S$. aureus), streptococci and gram-negative rods, for which any growth (i.e. $\geq 1 \mathrm{CFU} / \mathrm{mL}$ ) was considered positive (Trampuz et al., 2007). Of note, synovial fluid leukocyte count was not considered to be a diagnostic criterion within the first 6 weeks after surgery in inflammatory joint disease and in case of periprosthetic fracture or luxation. In these situations, the leukocyte count can be increased also in the absence of an infection (Renz et al., 2018b).

\section{Prosthesis retrieval}

Prosthetic components retrieved at revision surgery were separately placed into sterile containers. Then, the explanted material was transported within $4 \mathrm{~h}$ to the microbiology laboratory for sonication.

\section{Sonication of prosthesis components}

$100 \mathrm{~mL}$ sterile normal saline solution was added to each container for sonication. The implants were totally submerged in the fluid. Then, the samples were vortexed and sonicated according to a previously described protocol, using $40 \mathrm{kHz}$ and $0.2 \mathrm{~W} / \mathrm{cm}^{2}$ (Trampuz et al., 2007). $0.5 \mathrm{~mL}$ aliquots were plated on to tryptic soy agar with $5 \%$ sheep blood, chocolate agar and Schaedler $5 \%$ sheep blood agar, respectively; $3 \mathrm{~mL}$ aliquots were inoculated into thioglycolate broth (all from BioMedia, Saint Petersburg, Russia). Inoculations were done before sonication (i.e. vortexing culture) and after sonication (i.e. sonication culture). Aerobic and anaerobic sheepblood agar plates (BioMedia) were incubated for $14 \mathrm{~d}$ at $35{ }^{\circ} \mathrm{C}$ in $5 \% \mathrm{CO}_{2}$ aerobically and anaerobically, respectively. Cultures were quantified by counting the number of colonies that grew on the plate and adjusting to the number of CFU (CFU/mL). Identification and susceptibility testing of isolated microorganisms was performed using a WalkAway 96 Plus automatic bacteriological analyser (Beckman Coulter).

\section{Statistical analysis}

The sample size was calculated on the assumption that differences in retrieved biofilm by sonication is 
Table 1. Demographics and prostheses characteristics of 40 patients. ${ }^{1} \mathrm{All}$ made of polyethylene; ${ }^{2}$ all made of cobalt-chromium; ${ }^{3} 13$ made of titanium alloy, 3 made of polyethylene; ${ }^{4}$ all made of titanium alloy; ${ }^{5}$ all made of cobalt-chromium; ${ }^{6} 15$ made of titanium alloy, 2 made of cobalt-chromium alloy.

\begin{tabular}{|c|c|c|c|}
\hline Characteristics of the patients & All PJI $(n=40)$ & Knee PJI $(n=21)$ & Hip PJI $(n=19)$ \\
\hline Patient age, median (range) - years & $60(43-75)$ & $64(56-75)$ & $56(43-74)$ \\
\hline Male sex, number (\%) & $21(53)$ & $8(38)$ & $13(68)$ \\
\hline Number of components & 112 & 58 & 54 \\
\hline $\begin{array}{l}\text { Time from implantation until revision, } \\
\text { median (range) - months }\end{array}$ & $30.5(1.5-108)$ & $14.5(1.5-48)$ & $29(2-108)$ \\
\hline $\begin{array}{l}\text { Type of component, number } \\
\text { Liner }^{1} \\
\text { Femoral head }^{2} \\
\text { Acetabular cups }^{3} \\
\text { Femoral stems }^{4} \\
\text { Femoral knee components }^{5} \\
\text { Tibial components }^{6}\end{array}$ & $\begin{array}{l}29 \\
16 \\
16 \\
14 \\
20 \\
17\end{array}$ & $\begin{array}{c}21 \\
- \\
- \\
- \\
20 \\
17\end{array}$ & $\begin{array}{c}8 \\
16 \\
16 \\
14 \\
- \\
-\end{array}$ \\
\hline $\begin{array}{l}\text { Type of material, number } \\
\text { Titanium alloy } \\
\text { Cobalt-chromium alloy } \\
\text { Polyethylene }\end{array}$ & $\begin{array}{l}42 \\
38 \\
32\end{array}$ & $\begin{array}{l}15 \\
22 \\
21\end{array}$ & $\begin{array}{l}27 \\
16 \\
11\end{array}$ \\
\hline
\end{tabular}

15-20\% when different biomaterials are compared. To detect differences at a significance level of 0.05 (twosided) with power of $80 \%$, a total of 87 components are required. Statistical analyses were performed using SigmaPlot (version 13.0; Systat Software, Chicago, IL, USA), graphics were plotted using Prism software (version 7.03; GraphPad). Qualitative data are expressed as number of subjects and percentage. Quantitative data are presented as median and interquartile range To compare different groups, one-way ANOVA and Wilcoxon signed-rank test for independent samples were used. The significance level in hypothesis testing was predetermined at $p<0.05$.

\section{Results}

\section{Patient demographic data and prosthesis characteristics}

A total of 112 prosthetic components were retrieved from 40 patients with PJI affecting 21 knee and 19 hip arthroplasties (Table 1). 58 components (52\%) originated from knee prostheses and 54 components (48 \%) from hip prostheses. The median time from primary implantation until revision of the prosthesis was 30.5 months (range: $1-120$ months). The materials included 42 components made of titanium alloy, 38 made of cobalt-chromium alloy and 32 made of polyethylene. Not all components and/or materials were available for all patients.

\section{Microbial detection in sonication- and vortexing- fluid culture}

As required for inclusion in the study, prostheses retrieved from all 40 patients showed microbial growth in sonication- or vortexing-fluid culture from at least one prosthetic component. Out of 112 components, microorganisms were detected in 72 components (64\%) using the vortexing method and in 90 components (80\%) using the sonication method.

Fig. 1 shows the percentage of positive vortexing and sonication cultures according to the type of prosthesis components in knee and hip joints. Applying the sonication method, hip and knee polyethylene liners showed bacteria growth in $100 \%$ of components, followed by femoral components of the knee $(90 \%)$ and acetabular cups $(88 \%)$, while femoral head and stem showed the lowest positivity rate of $50 \%$. Vortexing method showed an inferior positivity rate than sonication for all components except for femoral heads and stems.

Fig. 2 shows the percentage of positive culture using vortexing and sonication methods stratified according to the type of biomaterial. Bacteria in sonication-fluid cultures grew in all polyethylene components, followed by titanium alloy (79\%) and cobalt-chromium (71\%). Compared to the vortexingonly method, additional positive cultures were found by using the sonication method in 4 implants made of titanium alloy ( $p=0.457), 6$ made of cobalt-chromium $(p=0.234)$ and 8 made of polyethylene $(p=0.005)$.

Fig. 3 shows the quantity of dislodged bacteria, stratified according to the type of prosthesis component of hip and knee prostheses. By the vortexing-only method, the median bacterial load was higher from hip and knee polyethylene liners (923 CFU/mL and $695 \mathrm{CFU} / \mathrm{mL}$, respectively) than from other prostheses components, but not significantly different $(p>0.05)$. By the sonication method, significantly higher median CFU/mL were found on hip liners (1250 CFU/mL) than on femoral 


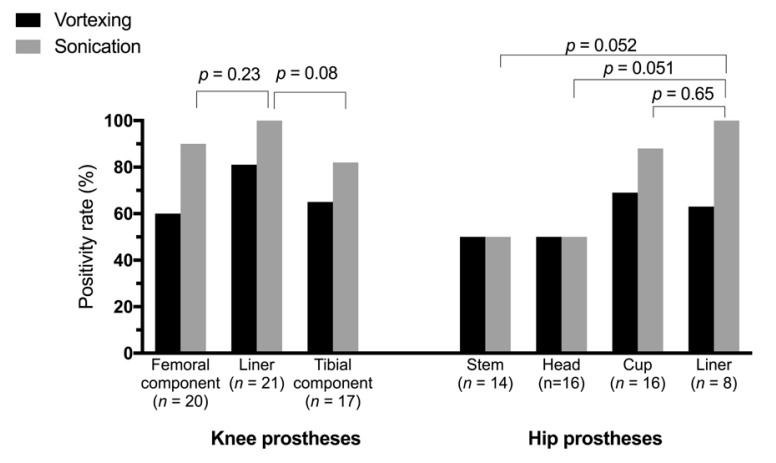

Fig. 1. Positivity rate of cultures after vortexing and sonication according to the type of component in knee and hip prostheses.

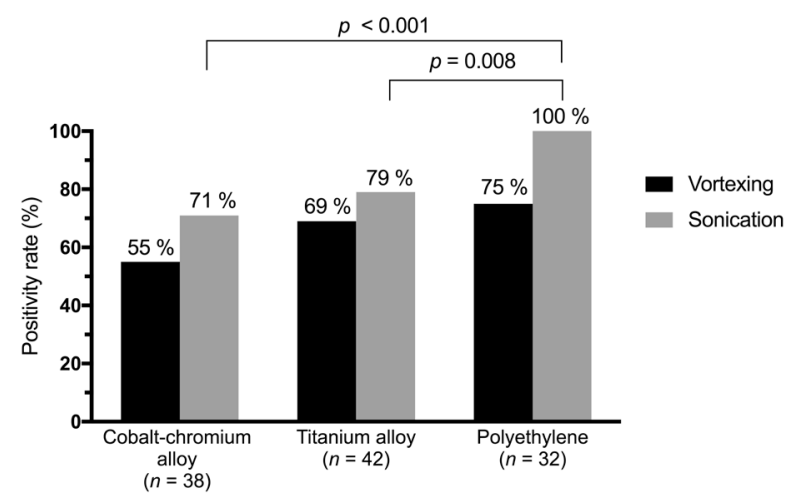

Fig. 2. Positivity rate of cultures after vortexing and sonication according to the type of material.
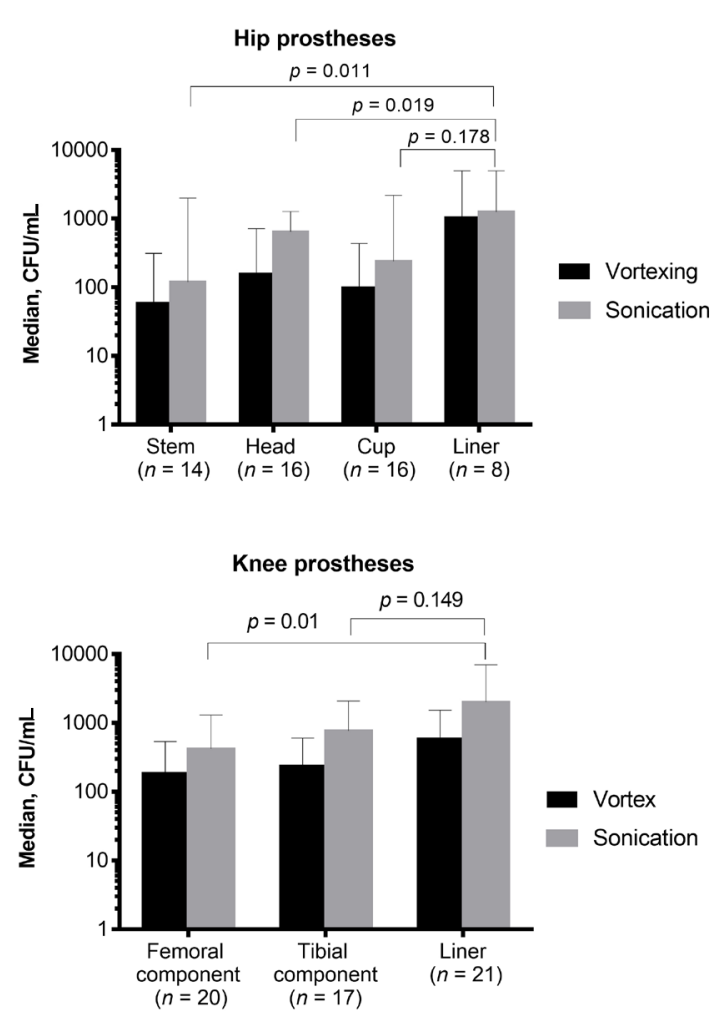

Fig. 3. Quantitative analysis of dislodged bacteria by vortexing and sonication $(\mathrm{CFU} / \mathrm{mL}$ ) in hip and knee prostheses according to the type of prosthesis component (median values are shown, error bars represent interquartile range). stems $(120 \mathrm{CFU} / \mathrm{mL}, p=0.011)$ or heads $(650 \mathrm{CFU} / \mathrm{mL}$, $p<0.019)$, but not on cups (270 CFU/mL, $p=0.178)$. In knees, polyethylene liners showed significantly higher median CFU/mL (2000 CFU/mL) than on femoral knee components (330 CFU/mL, $p=0.021$ ), but not on tibial components ( $800 \mathrm{CFU} / \mathrm{mL}, p=0.149$ ).

Fig. 4 shows the distribution of dislodged bacteria by vortexing and sonication methods stratified according to the different materials. The highest bacterial load was detected from polyethylene (by the sonication method), which was significantly higher than from any tested metal alloy (cobalt-chromium and titanium) by the sonication method $(p<0.05)$.

Qualitative and quantitative analysis of microorganisms

Coagulase-negative staphylococci were the most commonly isolated microorganism (mainly Staphylococcus epidermidis), followed by S. aureus and Streptococcus species. Table 2 shows the qualitative and quantitative microbiological results in vortexing and sonication cultures stratified according to pathogen. In sonication cultures, bacteria were more commonly detected than in vortexing cultures and the number of CFU was consistently larger. By sonication, coagulase-negative staphylococci were isolated from 51 out of 68 components (75\%), followed by S. aureus
( 27 out of 30 components, $90 \%$ ). The largest median CFU quantity in sonication culture was found with S. aureus $(1200 \mathrm{CFU} / \mathrm{mL})$, followed by coagulasenegative staphylococci (700 CFU/mL), Streptococcus species (680 CFU/mL), gram-negative bacteria (165 CFU/mL) and Enterococcus faecalis (100 CFU/mL).

\section{Discussion}

Previous studies show that sonication of removed implants considerably improve the diagnosis of PJI, especially in chronic, low-grade infections (Evangelopoulos et al., 2013; Holinka et al., 2011). In the present study, sonication detected additional microorganisms in $18 \%$ of the implants made of titanium, $11 \%$ of the implants made of cobaltchromium and $4 \%$ of the implants made of polyethylene, compared to the vortexing-only method. Large numbers of CFU grow in sonicationfluid cultures, as reported by Portillo et al. (2013).

Interestingly, the bacterial counts were larger after sonication of polyethylene liners than of metal alloys, as reported by Holinka et al. (2012). This observation demonstrated that the biomaterials had diverse intrinsic affinity for bacterial adhesion and biofilm formation. The removed biofilm 


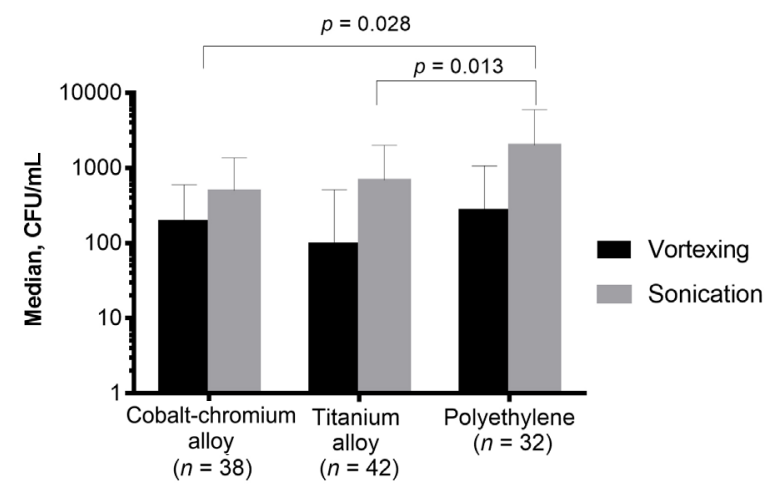

Fig. 4. Quantitative analysis of dislodged bacteria by vortexing and sonication $(\mathrm{CFU} / \mathrm{mL}$ ) according to the type of material (median values are shown, error bars represent interquartile range).

from retrieved implants was quantitatively and qualitatively evaluated, from which the total biofilm biomass originally present on the prosthesis surface was estimated, assuming equal sonication efficiency from different biomaterial types. However, differences in removed biofilms also reflected differences in removal efficiency by sonication from various types of materials. The inability to reliably determine actual biofilm biomass on the prosthetic component in situ before removal was an important limitation of the study (Holinka et al., 2012). All types of ex vivo investigations depend on several factors, including destruction of the biofilm during removal of the prosthetic components, fixation method used (cemented and non-cemented) and biomaterial surface type (i.e. roughness) (GomezBarrena et al., 2012). A further explanation for the inferior recovery of bacteria from sonicated cobaltchromium alloys as compared to polyethylene might be the toxic properties of metal-wear particles. Cobalt and chromium are found in toxicologically relevant concentrations in the tissue adjacent to metal-on-metal prostheses and their cytotoxic effect is confirmed in vitro. In vivo exposure to cobalt and chromium interferes with the capacity for osteogenic differentiation of mesenchymal stromal cells residing in the bone marrow (Rakow et al., 2016). The release of cobalt ions into the body is a key trigger of cell death, DNA damage and, subsequently, has been listed as a reasonably anticipated carcinogen (Schoon et al., 2017). However, the effect on bacterial viability on the prosthesis surface remains unclear.

Microbial characteristics - such as microorganism species, size of initial inoculum, interaction with the host defences, time elapsed from onset of adhesion to removal of the implants - further influence bacterial adhesion and biofilm formation (Gomez-Barrena et al., 2012). Also, microorganism species, maturity of biofilm, size and morphology of implants will affect sonication efficiency. However, sonication consistently demonstrated removal of $>99.9 \%$ of adherent bacteria from different materials, as demonstrated by scanning electron microscopy or confocal laser scanning imaging (data not shown). More reliable methods for biofilm quantification may be used to confirm the results of the study, including biofilm staining or metabolic detection assays (Magana et al., 2018).

Another limitation of the study was the impossibility of comparing biofilm quantity or quality on components in direct and forceful contact with bone and on those exposed to synovial fluid or soft tissue. In addition, significantly different surface features, such as treated, microrough or smooth surfaces, influence microbial adhesion (Cordero et al., 1994; Hussain et al., 2016), which was not analysed in the present study. Moreover, the polyethylene liners occupied a different space from the metals. If the liner were made of another material, they might have been more highly colonised because of the location rather than the material. This influence was not investigated in the study. A further limitation was the inability to calculate the exact component surface in relation to the fluid used for sonication. To standardise the study, a volume of $100 \mathrm{~mL}$ was chosen, as this represents the average of the volumes (50-200 mL) used by Gomez-Barrena et al. (2012) and Portillo et al. (2013). Finally, the indwelling time of the components was quite different and might further influence the microbial adhesion.

Despite the limitations, the study had important practical implications for future research and diagnostic routine praxis. If intrinsic differences in the ability for biofilm formation exist between various biomaterials, the present study might stimulate development of novel processes to use biomaterials with better microbial colonisation 'resistance' or to modify their surface accordingly. Modified surfaces might resist microbial colonisation and promote healing.

\section{Conclusions}

The bacterial counts were larger after sonication of polyethylene liners than of metal alloys, suggesting that intrinsic differences in the ability for biofilm formation existed among various biomaterials. Polyethylene components from hip and knee prostheses allowed the diagnosis of PJI in all investigated cases. These results suggested that sonication of retrieved polyethylene liners might be sufficient for detection of infecting pathogen in patients with chronic PJI, rather than submitting the whole prosthesis for sonication.

\section{Acknowledgements}

This work was supported by the PRO-IMPLANT Foundation (https://www.pro-implant-foundation. 
Table 2. Microbiological results of vortexing and sonication cultures of $\mathbf{1 1 2}$ retrieved components. Only significantly positive results were considered. The sum of vortexing and sonication cultures from the components exceed the total number of 112 due to polymicrobial infections. IQR: interquartile range.

\begin{tabular}{|l|c|c|c|c|c|}
\hline \multicolumn{1}{|c|}{$\begin{array}{c}\text { Type of } \\
\text { microorganism }\end{array}$} & $\begin{array}{c}\text { Number of } \\
\text { components }\end{array}$ & \multicolumn{2}{|c|}{ Vortexing culture } & \multicolumn{2}{c|}{ Sonication culture } \\
\hline & $\begin{array}{c}\text { Number of } \\
\text { components } \\
\text { with positive } \\
\text { culture (\%) }\end{array}$ & $\begin{array}{c}\text { Median, IQR } \\
\text { (CFU/mL) }\end{array}$ & $\begin{array}{c}\text { Number of } \\
\text { components } \\
\text { with positive } \\
\text { culture (\%) }\end{array}$ & $\begin{array}{c}\text { Median, IQR } \\
\text { (CFU/mL) }\end{array}$ \\
\hline $\begin{array}{l}\text { Coagulase-negative } \\
\text { staphylococci }\end{array}$ & 68 & $38(56)$ & $195(100-606)$ & $51(75)$ & $700(240-2000)$ \\
\hline S. aureus & 30 & $25(83)$ & $400(58-670)$ & $27(90)$ & $1200(300-3400)$ \\
\hline Streptococcus species & 9 & $7(78)$ & $400(350-600)$ & $9(100)$ & $680(233-1600)$ \\
\hline Enterococcus faecalis & 5 & $2(40)$ & $5(5-25)$ & $3(60)$ & $100(100-110)$ \\
\hline $\begin{array}{l}\text { Gram-negative } \\
\text { bacteria }\end{array}$ & 6 & $6(100)$ & $111(5-223)$ & $6(100)$ & $165(13-614)$ \\
\hline All pathogens & 118 & $78(66)$ & - & $96(81)$ & - \\
\hline
\end{tabular}

org), a no-profit organisation supporting research, education, global networking and care of patients with bone, joint or implant-associated infection, providing an unrestricted educational grant.

\section{References}

Akgun D, Perka C, Trampuz A, Renz N (2018) Outcome of hip and knee periprosthetic joint infections caused by pathogens resistant to biofilmactive antibiotics: results from a prospective cohort study. Arch Orthop Trauma Surg 138: 635-642.

Akgun D, Trampuz A, Perka C, Renz N (2017) High failure rates in treatment of streptococcal periprosthetic joint infection: results from a sevenyear retrospective cohort study. Bone Joint J 99-B: 653-659.

Cordero J, Munuera L, Folgueira MD (1994) Influence of metal implants on infection. An experimental study in rabbits. J Bone Joint Surg $\mathrm{Br}$ 76: 717-720.

Cordero J, Munuera L, Folgueira MD (1996) The influence of the chemical composition and surface of the implant on infection. Injury 27 Suppl 3: SC34-37.

Evangelopoulos DS, Stathopoulos IP, Morassi GP, Koufos S, Albarni A, Karampinas PK, Stylianakis A, Kohl S, Pneumaticos S, Vlamis J (2013) Sonication: a valuable technique for diagnosis and treatment of periprosthetic joint infections. ScientificWorldJournal 2013: 375140. DOI: 10.1155/2013/375140.

Font-Vizcarra L, Garcia S, Bori G, Martinez-Pastor JC, Zumbado A, Morata L, Mensa J, Soriano A (2012) Long-term results of acute prosthetic joint infection treated with debridement and prosthesis retention: a case-control study. Int J Artif Organs 35: 908-912.
Gomez-Barrena E, Esteban J, Medel F, MolinaManso D, Ortiz-Perez A, Cordero-Ampuero J, Puertolas JA (2012) Bacterial adherence to separated modular components in joint prosthesis: a clinical study. J Orthop Res 30: 1634-1639.

Holinka J, Bauer L, Hirschl AM, Graninger W, Windhager R, Presterl E (2011) Sonication cultures of explanted components as an add-on test to routinely conducted microbiological diagnostics improve pathogen detection. J Orthop Res 29: 617-622.

Holinka J, Pilz M, Hirschl AM, Graninger W, Windhager R, Presterl E (2012) Differential bacterial load on components of total knee prosthesis in patients with prosthetic joint infection. Int J Artif Organs 35: 735-741.

Hussain A, Curry B, Cahalan L, Minkin S, Gartner M, Cahalan P (2016) Development and in vitro evaluation of infection resistant materials: a novel surface modification process for silicone and Dacron. J Biomater Appl 30: 1103-1113.

Krenn V, Morawietz L, Perino G, Kienapfel H, Ascherl R, Hassenpflug GJ, Thomsen M, Thomas P, Huber M, Kendoff D, Baumhoer D, Krukemeyer MG, Natu S, Boettner F, Zustin J, Kolbel B, Ruther W, Kretzer JP, Tiemann A, Trampuz A, Frommelt L, Tichilow R, Soder S, Muller S, Parvizi J, Illgner U, Gehrke T (2014) Revised histopathological consensus classification of joint implant related pathology. Pathol Res Pract 210: 779-786.

Larsen LH, Lange J, Xu Y, Schonheyder HC (2012) Optimizing culture methods for diagnosis of prostheticjoint infections: a summary of modifications and improvements reported since 1995. J Med Microbiol 61: 309-316.

Lass R, Giurea A, Kubista B, Hirschl AM, Graninger W, Presterl E, Windhager R, Holinka J 
(2014) Bacterial adherence to different components of total hip prosthesis in patients with prosthetic joint infection. Int Orthop 38: 1597-1602.

Magana M, Sereti C, Ioannidis A, Mitchell CA, Ball AR, Magiorkinis E, Chatzipanagiotou S, Hamblin MR, Hadjifrangiskou M, Tegos GP (2018) Options and limitations in clinical investigation of bacterial biofilms. Clin Microbiol Rev 31. DOI: 10.1128/ CMR.00084-16.

Morgenstern C, Cabric S, Perka C, Trampuz A, Renz N (2018). Synovial fluid multiplex PCR is superior to culture for detection of low-virulent pathogens causing periprosthetic joint infection. Diagn Microbiol Infect Dis 90: 115-119.

Peel TN, Dylla BL, Hughes JG, Lynch DT, Greenwood-Quaintance KE, Cheng AC, Mandrekar JN, Patel R (2016) Improved diagnosis of prosthetic joint infection by culturing periprosthetic tissue specimens in blood culture bottles. MBio 7: e01776-15. DOI: 10.1128/mBio.01776-15.

Petty W, Spanier S, Shuster JJ, Silverthorne C (1985) The influence of skeletal implants on incidence of infection. Experiments in a canine model. J Bone Joint Surg Am 67: 1236-1244.

Portillo ME, Salvado M, Trampuz A, Plasencia V, Rodriguez-Villasante M, Sorli L, Puig L, Horcajada JP (2013) Sonication versus vortexing of implants for diagnosis of prosthetic joint infection. J Clin Microbiol 51: 591-594.

Puig-Verdie L, Alentorn-Geli E, Gonzalez-Cuevas A, Sorli L, Salvado M, Alier A, Pelfort X, Portillo ME, Horcajada JP (2013) Implant sonication increases the diagnostic accuracy of infection in patients with delayed, but not early, orthopaedic implant failure. Bone Joint J 95-B: 244-249.

Rakow A, Schoon J, Dienelt A, John T, Textor M, Duda G, Perka C, Schulze F, Ode A (2016) Influence of particulate and dissociated metal-on-metal hip endoprosthesis wear on mesenchymal stromal cells in vivo and in vitro. Biomaterials 98: 31-40.

Renz N, Feihl S, Cabric S, Trampuz A (2017) Performance of automated multiplex PCR using sonication fluid for diagnosis of periprosthetic joint infection: a prospective cohort. Infection 45: 877-884.
Renz N, Mudrovcic S, Perka C, Trampuz A (2018a) Orthopedic implant-associated infections caused by Cutibacterium spp. - A remaining diagnostic challenge. PLoS One 13: e0202639. DOI: 10.1371/ journal.pone.0202639.

Renz N, Yermak K, Perka C, Trampuz A (2018b) Alpha defensin lateral flow test for diagnosis of periprosthetic joint infection: not a screening but a confirmatory test. J Bone Joint Surg Am 100: 742-750.

Schoon J, Geißler S, Traeger J, Luch A, Tentschert J, Perino G, Schulze F, Duda GN, Perka C, Rakow A (2017) Multi-elemental nanoparticle exposure after tantalum component failure in hip arthroplasty: indepth analysis of a single case. Nanomedicine 13: 2415-2423.

Sigmund IK, Yermak K, Perka C, Trampuz A, Renz N (2018) Is the enzyme-linked immunosorbent assay more accurate than the lateral flow alpha defensin test for diagnosing periprosthetic joint infection? Clin Orthop Relat Res 476: 1645-1654.

Trampuz A, Piper KE, Jacobson MJ, Hanssen AD, Unni KK, Osmon DR, Mandrekar JN, Cockerill FR, Steckelberg JM, Greenleaf JF, Patel R (2007) Sonication of removed hip and knee prostheses for diagnosis of infection. N Engl J Med 357: 654-663.

\section{Discussion with Reviewer}

David Grainger: In vitro-in vivo correlations are a significant problem in this field. Your data would have allowed you to make the correlations with published in vitro data. Is this not a missed opportunity?

Authors: It is difficult to correlate in vitro with in vivo study results since the experimental conditions considerably influence the test result; therefore, the in vivo data were compared only with animal experimental studies (Cordero et al., 1994; Cordero et al., 1996; Petty et al., 1985).

Editor's note: The Scientific Editor responsible for this paper was Fintan Moriarty. 